\title{
Migraine and MTHFR C677T genotype in a population- based sample
}

\author{
Ann I. Scher, PhD $12 *$, Gisela M. Terwindt, MD, PhD 3, W. M. Monique \\ Verschuren, PhD 4, Mark C. Kruit, MD 5, Henk J. Blom, PhD 6, Hisanori \\ Kowa, MD, PhD 7, Rune R. Frants, PhD 8, Arn M. J. M. van den \\ Maagdenberg, PhD 3 8, Mark van Buchem, MD, PhD 5, Michel D. Ferrari, \\ MD, PhD 3, Lenore J. Launer, PhD 24
}

\author{
1Department of Preventive Medicine and Biometrics, Uniformed Services \\ University, Bethesda, MD \\ 2Laboratory of Epidemiology, Demography, and Biometry, National Institute on \\ Aging, National Institutes of Health, Bethesda, MD \\ 3Department of Neurology, Leiden University Medical Center, Leiden, The \\ Netherlands \\ 4Centre for Prevention and Health Services Research, National Institute of Public \\ Health and the Environment, Bilthoven, The Netherlands \\ 5Department of Radiology, Leiden University Medical Center, Leiden, The \\ Netherlands \\ 6Laboratory of Pediatrics and Neurology, Nijmegen Centre for Molecular Life \\ Sciences, Radboud University Nijmegen Medical Centre, The Netherlands \\ 7Department of Neurology, Institute of Neurological Sciences, Faculty of \\ Medicine, Tottori University, Yonago, Japan \\ 8Department of Human Genetics, Leiden University Medical Center, Leiden, The \\ Netherlands
}

email: Ann I. Scher (ascher@usuhs.mil)

* Correspondence to Ann I. Scher, Uniformed Services University, Department of Preventive Medicine and Biometrics, 4301 Jones Bridge Road; Bethesda, MD 20814-4799

\begin{abstract}
\section{Objective}

Migraine with aura is associated with increased risk of stroke. The MTHFR C677T genotype has been associated with increased risk of migraine in selected clinical samples and with elevated homocysteine, a risk factor for stroke. We assessed the association of the MTHFR C677T variant with migraine and the mediating effect of cardiovascular risk factors and metabolic markers of genotype status.
\end{abstract}

\section{Methods}

We compared adult migraineurs with aura (MA; $\mathrm{n}=187)$, without aura $(\mathrm{MO} ; \mathrm{n}=$ 226), and nonmigraineurs $(n=1,212)$ from the population-based Genetic Epidemiology of Migraine study.

\section{Results}

Compared with the wild-type genotype, the T/T genotype was associated with increased odds of MA (odds ratio [OR], 2.05; 95\% confidence interval, $1.2-3.4 ; \mathrm{p}$ $<0.006)$, with a trend of increasing numbers of T alleles (OR, $1.40 ; 95 \%$ confidence interval, 1.1-1.8; $\mathrm{p}<0.007)$. ORs were slightly attenuated after adjusting for homocysteine.

\section{Interpretation}

Risk of MA is associated with MTHFR C674T homozygosity, independent of other cardiovascular risk factors. Ann Neurol 2006 
Migraine sufferers, particularly those with aura, have an increased risk for clinical and subclinical vascular brain lesions.[1][2] The reason for this increased risk is unknown but does not appear to be caused by classic risk factors for cardiovascular (CV) disease.[2][3] Homocysteine, a sulfur-containing amino acid derived during the metabolism of the essential amino acid methionine, is a relatively new risk factor for CV disease. Elevated homocysteine or reduced folate (a cofactor in homocysteine metabolism) have been associated with various forms of ischemic vascular disease, endothelial dysfunction, and congenital defects of the neural tube and heart.[4][5]

The MTHFR gene on chromosome $1 p 36.3$ encodes 5,10methylenetetrahydrofolate reductase (MTHFR), a key enzyme in the metabolism of folate. A relatively common polymorphism in this gene, C677T, changes an alanine to a valine residue at position 222. Homozygotes (T/T) for this polymorphism have moderately increased plasma total homocysteine[6] and were reported, in some case-control studies, to have an increased risk of migraine with aura.[7-10] These studies, however, were conducted in selected clinical samples and did not control for CV risk factors.[3]

We examine the association between the MTHFR C677T genotype and migraine in an unselected, well-characterized cohort of men and women from a populationbased study. We also analyzed the effect of traditional CV risk factors and metabolic markers of the MTHFR C677T genotype status on this association.

\section{Subjects and Methods}

Study participants $(n=1,625)$ were an age and sex-stratified random sample from the Genetic Epidemiology of Migraine (GEM) study $(n=6,491)$. Participants for the GEM study were identified through the population-based MORGEN study (Monitoring Project on Risk Factors for Chronic Diseases in the Netherlands), that was based on an age-stratified random sample of adults, aged 20 to 65 years, living in two municipalities in the Netherlands.[11] All GEM participants received a detailed direct migraine diagnostic interview and other risk assessments as described previously,[3][12][13] including cardiovascular risk assessment.

Plasma total homocysteine, folate, and vitamin B12 were assayed in nonfasting plasma samples, and the MTHFR C677T variant was genotyped in 1,401 participants as described previously. [6] These included 1,212 individuals without migraine (44\% women), 117 with migraine without aura (MO; 79\% women), and 72 with migraine with aura (MA; 72\% women). An additional 224 migraineurs (MO 109; MA 115) were only genotyped, without metabolic measurements. Thus, in total, 1,625 participants were genotyped: 1,212 nonmigraineurs ( $44 \%$ women), 226 with MO (76\% women), and 187 with MA ( $73 \%$ women). The MA group consisted of 111 migraineurs with exclusively attacks with aura and 76 with both attacks with or without aura. At the time of measurement, there was no folic acid fortification of grains in the Netherlands.

\section{Analysis}

We determined whether observed genotype frequencies were in Hardy-Weinberg equilibrium. The Pearson's 2 test and Mantel-Haenzel test for trend were used to compare allele frequencies by categorical measures of cardiovascular risk. We used nonparametric methods (rank-sum test, quantile regression) for continuous measures of cardiovascular risk. We also used quantile regression (similar to linear regression) to calculate age and sex-adjusted median levels of homocysteine, folate, and vitamin B12. 
Multinomial logistic regression was used to calculate crude and adjusted odds ratios (ORs) as an estimate of the risk for MO and MA by genotype (C/T or T/T compared with those with the wild-type genotype $\mathrm{C} / \mathrm{C}$ ). Models were adjusted for age, sex, and socioeconomic status. We also included in the models cardiovascular risk factors and levels of homocysteine, folate, and vitamin B12 to determine whether these factors mediated the relationship between genotype and phenotype.

In secondary analyses, we examined whether the risk of migraine associated with the MTHFR C677T variant varied by age at onset of migraine ( $<10$ years, MO onset $10+$ years, MA onset $<10$ years, MA onset $10+$ year) or by frequency of attacks (MO $<12$ attacks/year, MO 12+ attacks/year, MA $<12$ attacks/year, MA $12+$ attacks/year). Analyses were performed using Stata (StataCorp. 2003. Stata Statistical Software: Release 8.2. College Station, TX: StataCorp LP).

\section{Results}

The frequency of the T allele in the nonmigraine group (31.5\%; Table 1 ) was consistent with the reported frequency of this allele in other white populations.[14] Allele frequencies were in Hardy-Weinberg equilibrium overall, and within all study subgroups.

Table 1. MTHFR C677T Genotype and T Allele Frequency by Migraine Status: Genetic Epidemiology of Migraine Study

\begin{tabular}{|c|c|c|c|c|c|}
\hline \multirow[b]{2}{*}{$\begin{array}{l}\text { Migraine } \\
\text { Status }\end{array}$} & \multicolumn{3}{|c|}{ Genotype } & \multirow[b]{2}{*}{$\begin{array}{c}\mathbf{T} \text { allele } \\
\text { Frequency }\end{array}$} & \multirow[b]{2}{*}{$\begin{array}{c}\text { Total }(n= \\
1,625)\end{array}$} \\
\hline & $\begin{array}{c}C / C(n= \\
748)\end{array}$ & $\begin{array}{c}C / T(n= \\
713)\end{array}$ & $\begin{array}{c}T / T(n= \\
164)\end{array}$ & & \\
\hline No migraine & $567(47 \%)$ & $527(43 \%)$ & $118(10 \%)$ & $31.5 \%$ & $1,212(100 \%)$ \\
\hline MO & $109(48 \%)$ & $100(44 \%)$ & $17(8 \%)$ & $29.6 \%$ & $226(100 \%)$ \\
\hline MA & $72(39 \%)$ & $86(46 \%)$ & $29(16 \%)$ & $38.5 \%$ & $187(100 \%)$ \\
\hline
\end{tabular}

$\mathrm{C} / \mathrm{C}=$ wild type $\mathrm{C} / \mathrm{T}=$ heterozygote $\mathrm{T} / \mathrm{T}=$ homozygote $; \mathrm{MO}=$ migraine without aura; $M A=$ migraine with aura.

Genotype status was associated with total homocysteine (higher in the $\mathrm{C} / \mathrm{T}$ and $\mathrm{T} / \mathrm{T}$ groups) and folate (lower in the $\mathrm{C} / \mathrm{T}$ and $\mathrm{T} / \mathrm{T}$ groups; Table 2 ) and marginally with socioeconomic status (SES; lower for C/T $[p<0.08]$ and T/T $[p<0.06]$ with a significant trend overall for low SES and number of T alleles $[p<0.05]$ ). 
Table 2. Characteristics of the Study Sample by MTHFR C677T Genotype: Genetic Epidemiology of Migraine Study

\begin{tabular}{|c|c|c|c|}
\hline \multirow{2}{*}{$\begin{array}{l}\text { Characteristic } \\
\text { Sex (\% female })\end{array}$} & \multicolumn{3}{|c|}{$C / C(n=748) C / T(n=713) T / T(n=164)$} \\
\hline & 52.4 & 51.1 & 50.6 \\
\hline $\begin{array}{l}\text { Low SES }(\%)^{a} \\
\text { Maternal MI }(\%)\end{array}$ & 45.4 & 49.9 & 53.4 \\
\hline No & 90.5 & 90.4 & 90.4 \\
\hline Early (<65 yr) & 3.8 & 4.6 & 7.1 \\
\hline Late $(\geq 65 \mathrm{yr})$ & 5.7 & 5.0 & 2.6 \\
\hline \multicolumn{4}{|l|}{ Paternal MI (\%) } \\
\hline No & 75.0 & 77.2 & 71.8 \\
\hline Early (<55 yr) & 7.4 & 8.0 & 9.0 \\
\hline Late $(\geq 55$ yr $)$ & 17.6 & 14.7 & 19.2 \\
\hline CHD or stroke $(\%)^{\mathbf{b}}$ & 1.9 & 2.2 & 3.7 \\
\hline \multicolumn{4}{|c|}{ Mean (SD) values by genotype } \\
\hline Age & $41.3(11.8)$ & $41.6(11.5)$ & $41.6(11.3)$ \\
\hline Cholesterol (mg/dl) & $200(41)$ & $200(41)$ & $204(42)$ \\
\hline $\mathrm{HDL}(\mathrm{mg} / \mathrm{dl})$ & $51.2(14.3)$ & $51.7(13.8)$ & $51.1(11.3)$ \\
\hline Total: HDL & $4.2(1.5)$ & $4.1(1.5)$ & $4.2(1.1)$ \\
\hline Waist hip ratio & $0.84(0.09)$ & $0.85(0.09)$ & $0.85(0.09)$ \\
\hline Body mass index & $25.5(4.2)$ & $25.3(3.9)$ & $25.5(4.0)$ \\
\hline Systolic BP (mm Hg) & $121.8(15.3)$ & $121.8(15.0)$ & $122.9(15.3)$ \\
\hline \multirow[t]{2}{*}{ Diastolic BP $(\mathrm{mm} \mathrm{Hg})$} & $78.6(10.3)$ & $78.2(10.1)$ & $79.8(10.3)$ \\
\hline & $C / C(n=651)$ & $C / T(n=609)$ & $\mathrm{T} / \mathrm{T}(\mathrm{n}=141)$ \\
\hline Folate & $9.3(4.9)$ & $8.6(5.0)^{d}$ & $7.7(4.6)^{d}$ \\
\hline Homocysteine $\left(\boldsymbol{\mu}_{\mathrm{mol} / \mathrm{L}}\right)$ & $12.9(3.1)$ & $13.8(4.7)^{d}$ & $18.0(9.9)^{d}$ \\
\hline Vitamin $\mathrm{B}_{12}(\mathrm{nmol} / \mathrm{L})$ & $300(115)$ & $297(115)$ & $297(121)$ \\
\hline
\end{tabular}

a $\mathrm{p}<0.05 \mathrm{M}-\mathrm{H}$ test for trend for number of $\mathrm{T}$ alleles.

b Defined as self-reported history of myocardial infarction (MI), stroke, coronary bypass surgery, or balloon angioplasty.

c Sample is 1,625 except for levels of folate, homocysteine, vitamin B12 when sample size is $\mathrm{n}=1401$.

$\mathrm{d} p<0.01$. rank sum test compared to $\mathrm{c} / \mathrm{c}$ genotype; $\mathrm{p}<0.001$ test for trend for number of $\mathrm{T}$ alleles.

Compared with those with the wild-type genotype, those with the T/T genotype were at significantly increased risk of MA but not MO (Table 3). There was a significant trend for the number of T alleles and MA, but not MO (see Table 3, trend). ORs did not change after adjustment for age, sex, and SES (see Table 3, model 2) or additional adjustment for traditional cardiovascular risk factors (see Table 3, model 3). Further adjustment for plasma folate and vitamin B12 levels did not modify the association between MA and the MTHFR C677T variant. However, the association between genotype and MA was slightly attenuated and lost significance after adjustment for total homocysteine (from OR 2.35, p < 0.026 to OR 2.16, $\mathrm{p}<0.057$ ). In secondary analyses (Online Supplementary Table S1), results were similar when migraineurs were classified by age at onset or attack frequency. 
Table 3. Association between MTHFR C677T Genotype and Migraine without Aura $(M O, n=226)$ and Migraine with Aura (MA, $n=187)$

\begin{tabular}{|c|c|c|c|}
\hline \multirow{2}{*}{\multicolumn{2}{|c|}{$\mathbf{N}$}} & \multicolumn{2}{|c|}{ Migraine Classification } \\
\hline & & MO $(n=226)$ & MA $(n=187)$ \\
\hline \multicolumn{4}{|c|}{ Unadjusted OR } \\
\hline $\mathrm{C} / \mathrm{C}$ & 748 & 31.00 & 1.00 \\
\hline $\mathrm{C} / \mathrm{T}$ & 713 & $30.99(C$ & $1.29(0$ \\
\hline $\mathrm{T} / \mathrm{T}$ & 164 & $40.75(0.4-1.3)$ & $1.94(1.2-3.1)^{a}$ \\
\hline Trend & & $0.92(0.7-1.1)$ & $1.37(1.1-1.7)^{\mathrm{a}}$ \\
\hline \multicolumn{4}{|c|}{ Adjusted for age, sex, SES ${ }^{\mathbf{b}}$} \\
\hline $\mathrm{C} / \mathrm{C}$ & 748 & 81.00 & 1.00 \\
\hline $\mathrm{C} / \mathrm{T}$ & 713 & $31.00(C$ & $1.30(0.9-1.8)$ \\
\hline $\mathrm{T} / \mathrm{T}$ & 164 & $40.79(0$ & $2.02(1.2-3.3)^{a}$ \\
\hline Trenc & & $0.93(0.7-1.2)$ & $1.39(1.1-1.8)^{\mathrm{a}}$ \\
\hline \multicolumn{4}{|c|}{+ CVD risk factors ${ }^{\mathrm{c}}$} \\
\hline $\mathrm{C} / \mathrm{C}$ & 748 & 81.00 & 1.00 \\
\hline $\mathrm{C} / \mathrm{T}$ & 713 & $30.99(($ & $1.29(0$. \\
\hline $\mathrm{T} / \mathrm{T}$ & 164 & $40.79(0.4-1.4)$ & $2.05(1.2-3.4)^{a}$ \\
\hline
\end{tabular}

Comparison group consists of 1,212 individuals without migraine. ORs from multinomial logistic regression are adjusted as indicated. The outcome variable is migraine status (nonmigraine, $\mathrm{MO}, \mathrm{MA}$ ) and the independent variable is genotype $(C / C, C / T, T / T)$. Trend estimates odds of $M O$ or $M A$ with each additional $T$ allele compared with the odds of MO or MA in the C/C genotype.

a $\mathrm{p}<0.01$.

b Adjusted for age $\times$ sex, age-squared, socioeconomic status.

c Adjusted for above + waist-hip ratio, body mass index, smoking, total cholesterol, $\mathrm{HDL}$, total: $\mathrm{HDL}$ ratio, systolic BP, diastolic $\mathrm{BP}$, alcohol consumption. $\mathrm{C} / \mathrm{C}=$ wild type; $\mathrm{C} / \mathrm{T}=$ heterozygote $; \mathrm{T} / \mathrm{T}=$ homozygote $; \mathrm{MO}=$ migraine without aura; $M A=$ migraine with aura.

Overall, there were no differences in age-adjusted median levels of homocysteine, folate, and vitamin B12 among MO, MA, and nonmigraineurs (complete data are shown in Online Supplementary Tables S2 and S3 with the exception of one subgroup. The men in the MA T/T subgroup had the highest ageadjusted median levels of homocysteine $(22.9$ [20.3-25.6] $\mathrm{mol} / \mathrm{L}$ ) and lowest adjusted median levels of folate $(3.2[0.9-5.5] \mathrm{nmol} / \mathrm{L})$ compared with all other groups $(p<0.05$ test for interaction by sex).

\section{Discussion}

In this population-based sample, individuals with the MTHFR T/T genotype were at approximately two times the risk for MA compared with individuals with the wild-type CC genotype. The association was not mediated by cardiovascular risk factors or by plasma levels of two B vitamins that are involved in homocysteine metabolism, although there was a marginal indication that levels of homocysteine may partially mediate the association. We found no association between MO and genotype or metabolic markers.

Several clinic based case-control studies reported the association of migraine and the MTHFR C677T variation, but results have been inconsistent.[7-10] Kowa and colleagues[7] found an association between MA and the T/T genotype (OR $=6.5$; 95\% confidence interval [CI], 2.5-16.8) in Japanese patients. Kara and colleagues[9] did not find such an association in Turkish patients, although they 
did find an association between migraine overall and the $\mathrm{T} / \mathrm{T}$ genotype (OR $=5.7$; $95 \% \mathrm{CI}, 1.1-27.5)$. Oterino and colleagues[10] failed to find the T/T genotype to be associated with Spanish migraineurs, although the TT genotype was more common in those with aura compared with those without aura (OR, 2.34; 95\% CI, 1.0-5.3). Finally, Lea and colleagues[8] reported significant associations between MA and the T/T genotype both in familial (OR, 1.9; $95 \% \mathrm{CI}, 1.0-3.5$ ) and unrelated $(\mathrm{OR}=2.5 ; 95 \% \mathrm{CI}, 1.4-4.7)$ Australian migraineurs. These earlier studies have important limitations. All were from selected populations (eg, clinicbased patient samples or from a migraine pedigree family[8]). Furthermore, other than the migraine pedigree family study, studies did not describe the source of the control group, so it therefore is unclear whether the controls are from the same population as the patients.

We found higher homocysteine and lower folate levels in only one subgroup (male $\mathrm{T} / \mathrm{T}$ homozygotes with MA). We are not aware of any other studies of homocysteine and migraine from population samples; three clinic-based studies that compared homocysteine levels in migraine patients to controls produced conflicting results. [15-17]

Strengths of this study include our large, well-characterized, population-based cohort. Our migraine sufferers represent a range of severity and not just the presumably more severe phenotype seen in subspecialty care. Our migraine diagnosis was based on standardized diagnostic criteria and was supervised by neurologists who are experts in headache. Migraine with aura was diagnosed after a careful clinical history, which included subjects' drawings of their visual symptoms, thus minimizing the risk of misinterpreting visual symptoms. We also had measures of CV risk factors and, for a large subsample of our population, measurement of metabolic markers of the MTHFR C677T variant that might mediate the relationship between genotype and phenotype. However, we note that our measurement of these plasma biomarkers was from one adult blood draw and thus only represents a snapshot of lifetime exposure. Furthermore, homocysteine levels can be influenced by the presence of subclinical and clinical cardiovascular disease, other disease states, and certain medications that we could not account for in our analyses. [4] In addition, we did not measure the biomarkers in the entire study sample.

The mechanism by which the MTHFR C677T variant might increase the risk of MA (but not MO) is uncertain, although its effect on homocysteine and folate are plausible mechanisms. Increased homocysteine or reduced folate have been associated with disruption of endothelial function, which may be specifically relevant to cortical spreading depression, the presumed mechanism behind the migraine aura.[18-20] Our finding that the association was only slightly attenuated after adjusting for total homocysteine argues against this hypothesis, although limitations of our homocysteine measurement are noted above.

These results provide the first evidence in a population-based sample of an association between a specific genetic variant and a common migraine type. Future study is warranted to explore the possible influence of homocysteine and folate levels on MA.

\section{Acknowledgements}

This study was supported by the Ministry of Health, Welfare and Sport of the Netherlands and the National Institute of Public Health and the Environment [MDF, AMJMvdM, RRF, MV], the European Community EUROHEAD (LSHM-CT2004-504837) [MDF, AMJMvdM], VICI-grant Dutch Organisation for Scientific Research (NOW, Vici 918.56.602, MDF], Center of Medical System Biology 
(CMSB) established by the Netherlands Genomics Initiative/Netherlands Organisation for Scientific Research (NGI/NWO) [RRF, MDF], the Dutch Heart Association [MB, LL, MDF], and the NIH (National Institute on Aging Intramural Research Program, AS, LL].

Some of the results of this study were presented at the American Neurological Association 129th annual meeting, Toronto, Ontario, Canada, 10/04.

\section{References}

1 Swartz RH, Kern RZ. Migraine is associated with magnetic resonance imaging white matter abnormalities: a meta-analysis. Arch Neurol 2004; 61: 1366-1368.

2 Kruit MC, van Buchem MA, Hofman PA, et al. Migraine as a risk factor for subclinical brain lesions. JAMA 2004; 291: 427-434.

3 Scher AI, Terwindt GM, Picavet HSJ, et al. Cardiovascular risk factors and migraine: the GEM population-based study. Neurology 2005; 64: 614-620.

4 De Bree $A$, Verschuren WM, Kromhout $D$, et al. Homocysteine determinants and the evidence to what extent homocysteine determines the risk of coronary heart disease. Pharmacol Rev 2002; 54: 599-618.

5 Hobbs CA, Cleves MA, Melnyk S, et al. Congenital heart defects and abnormal maternal biomarkers of methionine and homocysteine metabolism. Am J Clin Nutr 2005; 81: 147-153.

6 de Bree A, Verschuren WM, Bjorke-Monsen AL, et al. Effect of the methylenetetrahydrofolate reductase $677 \mathrm{CT}$ mutation on the relations among folate intake and plasma folate and homocysteine concentrations in a general population sample. Am J Clin Nutr 2003; 77: 687-693.

7 Kowa H, Yasui K, Takeshima T, et al. The homozygous C677T mutation in the methylenetetrahydrofolate reductase gene is a genetic risk factor for migraine. Am J Med Genet 2000; 96: 762-764.

8 Lea RA, Ovcaric M, Sundholm J, et al. The methylenetetrahydrofolate reductase gene variant C677T influences susceptibility to migraine with aura. BMC Med 2004; 2: 3.

9 Kara I, Sazci A, Ergul E, et al. Association of the C677T and A1298C polymorphisms in the 5,10 methylenetetrahydrofolate reductase gene in patients with migraine risk. Brain Res Mol Brain Res 2003; 111: 84-90.

10 Oterino A, Valle N, Bravo $\mathrm{Y}$, et al. MTHFR T677 homozygosis influences the presence of aura in migraineurs. Cephalalgia 2004; 24: 491-494.

11 Picavet HS, Schouten JS, Smit HA. Prevalence and consequences of low back problems in the Netherlands, working vs non-working population, the MORGEN-Study. Monitoring Project on Risk Factors for Chronic Disease. Public Health 1999; 113: 73-77.

12 Launer LJ, Terwindt GM, Ferrari MD. The prevalence and characteristics of migraine in a population-based cohort: the GEM study. Neurology 1999; 53: 537-542.

13 Terwindt GM, Ferrari MD, Tijhuis M, et al. The impact of migraine on quality of life in the general population: the GEM study. Neurology 2000; 55: 624-629.

14 Botto LD, Yang Q. 5,10-Methylenetetrahydrofolate reductase gene variants and congenital anomalies: a HuGE review. Am J Epidemiol 2000; 151: 862-877.

15 Hering-Hanit R, Gadoth N, Yavetz A, et al. Is blood homocysteine elevated in migraine? Headache 2001; 41: 779-781.

16 Evers, S, Koch HG, Suhr B, Husstedt IW. Increased homocysteine level in migraine with aura. Eur J Neurol 1998; 5( suppl 3): S51-S52.

17 D'Amico D, Moschiano F, Attanasio A, et al. Hyperhomocysteinemia in patients with migraine with aura. Cephalalgia 2003; 23: 742.

18 Dreier JP, Kleeberg J, Petzold G, et al. Endothelin-1 potently induces Leao's cortical spreading depression in vivo in the rat: a model for an endothelial trigger of migrainous aura? Brain 2002; 125 : 102-112.

19 Splaver A, Lamas GA, Hennekens $\mathrm{CH}$. Homocysteine and cardiovascular disease: biological mechanisms, observational epidemiology, and the need for randomized trials. Am Heart J 2004; 148: 34-40.

20 Hadjikhani N, Sanchez dR, Wu O, et al. Mechanisms of migraine aura revealed by functional MRI in human visual cortex. Proc Natl Acad Sci USA 2001; 98: 4687-4692. 